\title{
Long-term outcome of electrical cardioversion in patients with chronic atrial flutter
}

Harry J G M Crijns, Isabelle C Van Gelder, Robert G Tieleman, Johan Brügemann, Pieter J De Kam, A T Marcel Gosselink, Margreet Th E Bink-Boelkens, Kong I Lie

\begin{abstract}
Objective-To determine the long-term outcome of serial electrical cardioversion therapy in patients with chronic atrial flutter.

Design-Prospective study, case series. Setting-University hospital.

Patients-50 consecutive patients with chronic ( $>24$ hours) atrial flutter without a previous relapse on antiarrhythmic drugs.

Interventions-Elective electrical cardioversion therapy, if necessary repeated, to obtain and keep patients in sinus rhythm. If the first cardioversion resulted in sinus rhythm, patients were not given antiarrhythmic drugs. Relapses were managed by repeated cardioversions then antiarrhythmic drugs were used serially in a set sequence.
\end{abstract}

Main outcome measure-Maintenance of sinus rhythm.

Results-Mean (SD) follow up was 3.5 $(1 \cdot 7)$ years. The first cardioversion was successful in 48 patients $(96 \%)$. After a single shock and without antiarrhythmic drugs being used, $42 \%$ of the patients maintained sinus rhythm in the longterm. Only left atrial size was inversely related to the efficacy of one shock $(P=$ $0 \cdot 025$ ). With serial cardioversion $90 \%$ of the patients were kept in sinus rhythm for 5 years. Univariate analysis showed that a long duration of arrhythmia and impaired cardiac function were both related to poor outcome. During follow up 3 patients died of progression of heart failure and another 5 died suddenly. None of these 5 patients was on antiarrhythmic drugs.

Conclusions-Electrical cardioversion was an effective and safe method of converting chronic atrial flutter to sinus rhythm. To maintain sinus rhythm, more than half of the patients required multiple shocks and prophylactic antiarrhythmic drugs. Sudden death was relatively frequent in the study population; the limited data available from this study suggest that such deaths were caused by the underlying disease and not drug related proarrhythmia.

(Heart 1997;77:56-61)

Keywords: atrial flutter; electrical cardioversion; mortality; congestive heart failure.
Restoration of sinus rhythm using direct current electrical cardioversion in patients with chronic atrial flutter is often successful. ${ }^{2}$ Subsequent arrhythmia suppression is thought to be relatively difficult. However, this assumption is based on limited data from patients with atrial flutter in the presence of (corrected) congenital heart diseases. ${ }^{3}$ Currently, the substrate of a typical atrial flutter is attributed to a right atrial macroreentrant circuit. ${ }^{4-6}$ This prompted electrophysiologists to attempt radiofrequency catheter ablation of this arrhythmia. The initial success rate of $90 \%$ was encouraging but the arrhythmia recurrence rate was 15 to $40 \%$ during 1.5 to 5 years follow up. Relapses of the arrhythmia seem predominantly related to the severity of the underlying heart disease..$^{7-11}$ However, these findings should be viewed against the background of the conservative usage of electrical cardioversion therapy. The aim of the present study was to investigate prospectively the long-term outcome of electrical cardioversion using a serial cardioversion approach and to determine factors that predict its success.

Patients and methods

PATIENT CHARACTERISTICS

Between February 1986 and January 1993101 consecutive patients with chronic atrial flutter were referred to our hospital. Criteria for chronicity of atrial flutter were at least two electrocardiographic documentations and continuous presence of flutter on a 24 hour Holter recording. Atrial flutter was subdivided into common or uncommon types. ${ }^{11-13}$ Common atrial flutter (type I) is characterised by negative sawtooth-like flutter waves in surface electrocardiographic leads II and III that are extremely consistent. In these inferior leads, there is a gentle downslope leading to a negative wave, followed by a sharp upstroke that overshoots slightly, before the start of the downsloping segment of the next cycle. Uncommon atrial flutter (type II) shows, also very consistently, positive flutter waves in the inferior leads. The atrial rate in common atrial flutter is between 240 and 340 beats per minute. In contrast, in uncommon atrial flutter the frequency is between 340 and 430 beats per minute. Neither age nor previous arrhythmia duration were exclusion criteria for participation in the study. Atrial fibrillation was never documented in these patients. We excluded from cardioversion patients with severe exercise limitation which was equivalent to grade IV on the New York Heart 

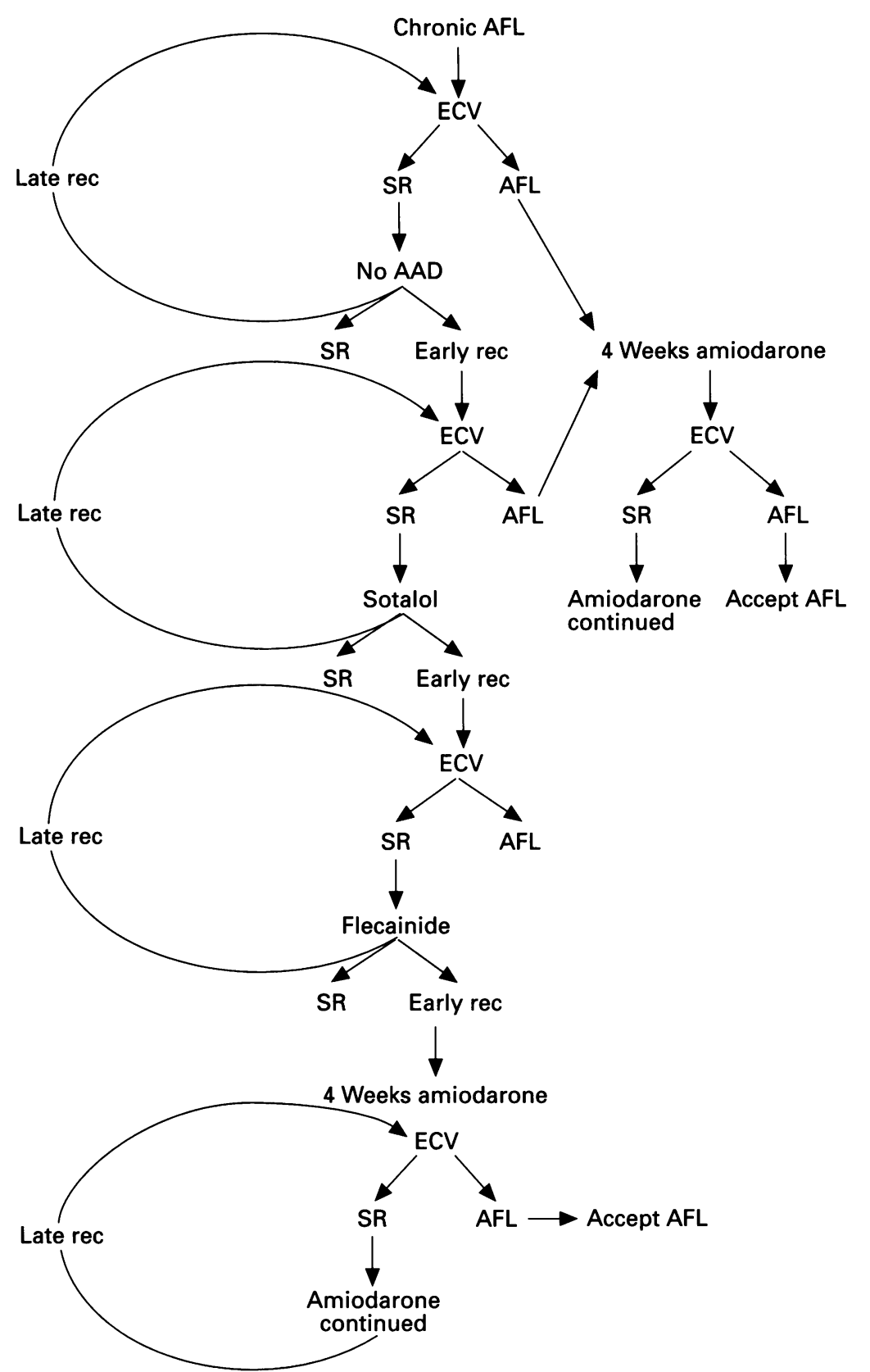

Figure 1 Flow chart of the serial electrical cardioversion (ECV) protocol. Patients with late recurrences (that is, after one year of sinus rhythm (SR)) underwent re-cardioversions without postshock drug prophylaxis or without a change in the antiarrhythmic drug that was being used. Patients who had contraindications for an antiarrhythmic drug (AAD) proceeded to the next stage. Verapamil was always started at the same time as flecainide to prevent rapid ventricular rates in the event of a recurrence of atrial flutter (AFL). Early rec, recurrence within one year; late rec, recurrence after one year.

Association classification scale $(\mathrm{n}=10)$, patients with unstable angina pectoris $(\mathrm{n}=$ 5 ), or an acute myocardial infarction less than four weeks ago $(n=3)$. Also not included were patients with a relapse of chronic atrial flutter while receiving an antiarrhythmic drug $(n=33)$. This left 50 patients for inclusion in the study. Part of this group has been described previously. ${ }^{214} 15$

TREATMENT

Elective electrical cardioversion was the principal treatment for atrial flutter. After the first cardioversion no antiarrhythmic drug treatment was used. After a relapse of the arrhythmia our serial prophylactic antiarrhythmic drug strategy was applied (fig 1). In case of failure to reestablish sinus rhythm, the rate control alternative was adopted. The protocol was approved by the ethics committee of our hospital and published elsewhere. ${ }^{2}$ In short, to prevent thromboembolic complications patients with atrial flutter were treated in the same way as patients with atrial fibrillation. Patients received warfarin or a derivative at least four weeks before the procedure. The target prothrombin time was $2 \cdot 4-4 \cdot 8$ international normalised ratio (INR). On the day of electrical cardioversion a two-dimensional transthoracic echocardiographic examination was done. During this procedure left and right atrial dimensions and left ventricular dimensions were determined according to the method of Schiller et al. ${ }^{16}$ Electrical cardioversion was performed without antiarrhythmic drug pretreatment in the postabsorptive state during light general anaesthesia with $20 \mathrm{mg}$ of intravenous etomidate. Two investigators (ICVG and ATMG) managed all procedures at the coronary care unit. A calibrated Hewlett Packard 43120-A defibrillator that can store 360 Joules of energy was used as the cardioverter device. One paddle was placed in the second intercostal space right parasternally, the other was placed in a left lateral position along the midaxillary line. According to the protocol, the first shock consisted of 30 Joules. Thereafter, the energy load of successive shocks was doubled until sinus rhythm was restored or after two attempts with 360 Joules. Post-shock rhythm monitoring was done by telemetry for 8-24 hours. Successful cardioversion was defined as maintenance of sinus rhythm more than eight hours after cardioversion.

After the first successful cardioversion patients were not treated with antiarrhythmic drugs prophylactically. All patients were seen at one, three, and six months after discharge at the outpatient department. Thereafter patients were reviewed every six months. Anticoagulants were discontinued if sinus rhythm persisted for more than one month after its restoration, except in those who required prolonged anticoagulation-for example, in cases of mitral stenosis. If atrial flutter relapsed, patients underwent repeated electrical cardioversion as soon as possible. Those who clearly felt that the arrhythmia lasted less than 24 hours were subject to cardioversion without preceding anticoagulation, the others were given warfarin for at least one month.

Patients with successive arrhythmia recurrences, were treated consecutively with three different prophylactic antiarrhythmic drugs. Until 1989 we used flecainide as the initial agent but after the results of the CAST trial ${ }^{17}$ became known our first preference was sotalol (160-320 mg daily), then flecainide (200$300 \mathrm{mg}$ daily), and finally amiodarone ( $600 \mathrm{mg}$ daily for four weeks followed by 200-300 mg daily). Amiodarone was started $\geqslant$ four weeks before a repeated electrical cardioversion. Patients with a relapse of atrial flutter after more than one year in sinus rhythm, a so called late recurrence, underwent electrical cardioversion without subsequent 
Table 1 Characteristics of patients with a successful single cardioversion approach compared with patients in whom this approach failed

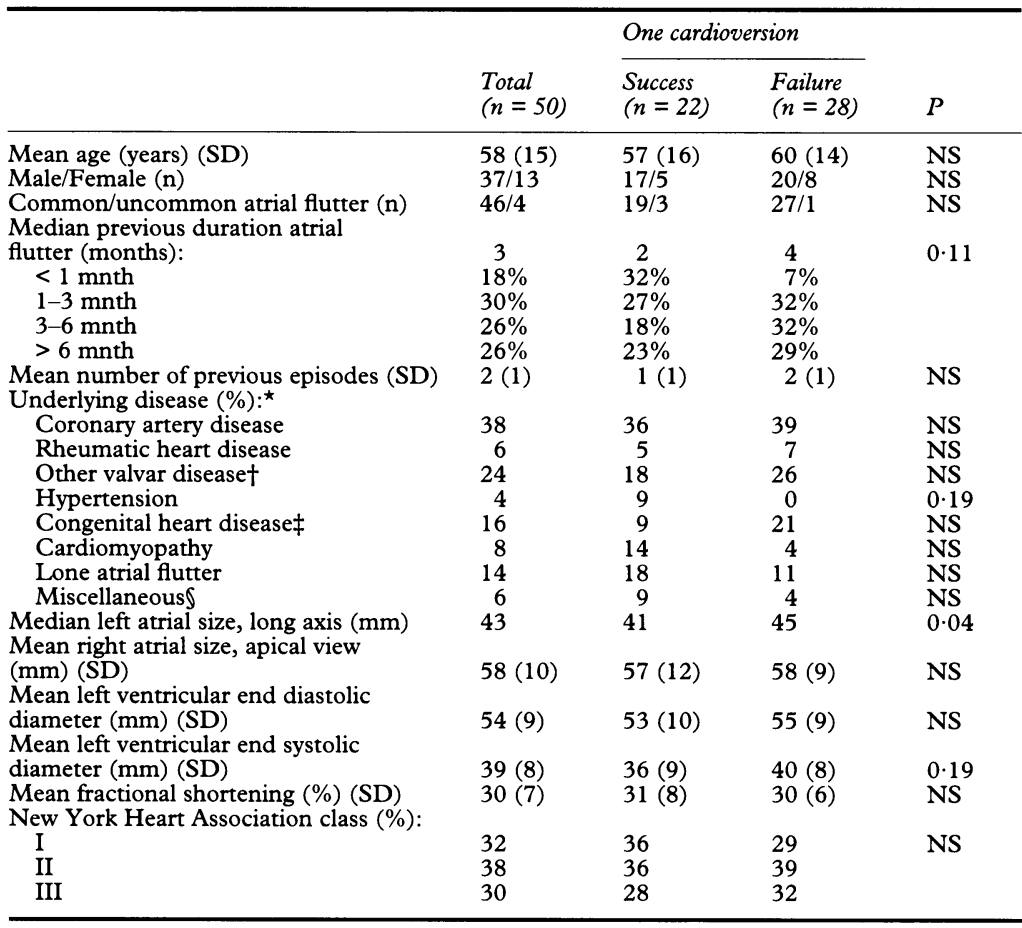

* More than one underlying disease per patient is scored. † Atherosclerotic mitral regurgitation, four patients (one mitral valve replacement); mitral regurgitation in the presence of dilated cardiomyopathy, one patient; aortic regurgitation, four patients (one aortic valve replacement); aortic stenosis, three patients (two aortic valve replacement); $\ddagger$ includes patients after repair of an ostium secundum atrial septum defect $(n=4)$, patients after correction of tetralogy of Fallot $(n=2)$, patients with repair of coarctation of the aorta $(n=1)$, and patients with repair of a $(\mathrm{n}=2)$, patients with repair of coarctation of the aorta $(\mathrm{n}=1)$, and patients with repair of a
persisting ductus Botalli $(\mathrm{n}=1)$; $\$ includes two patients with a sick sinus syndrome treated with persisting ductus Botali $(\mathrm{n}=1)$; includes two patients with a sick
artificial pacemaker and one patient after removal of a myxoma.

prophylactic antiarrhythmic drug treatment or without change of the antiarrhythmic drug eventually used. Patients in whom a certain antiarrhythmic drug was contraindicated continued to the next agent in our sequence.

The inclusion date was the day of the first cardioversion and follow up was completed by death or at 1 January 1994, whichever came first. Atrial flutter was accepted as inevitable if amiodarone was unsucessful, if there were drug related side effects, if completely asymptomatic arrhythmia was experienced after cardioversion(s), or if the patient refused another electrical cardioversion. In these patients, ventricular rate control of atrial flutter was attempted with digitalis and if necessary with additional verapamil, diltiazem, or a $\beta$ blocker with the aim of obtaining a resting heart rate under 100 beats $/ \mathrm{min}$. His bundle ablation with implantation of a pacemaker was offered to the patient if palpitations were severe or if there was progression or persistence of tachycardia related heart failure.

CARDIOVASCULAR EVENTS DURING FOLLOW UP The events that we monitored were recurrence of atrial flutter, incidence of thromboembolic complications and bleeding complications, progression of congestive heart failure, and antiarrhythmic drug related adverse events.

Intraventricular conduction delay was defined as a prolongation of the QRS duration $>50 \%$ of the baseline value. Ventricular proarrhythmia was defined as a fourfold increase in the number of ventricular premature beats, a tenfold increase in the number of couplets and non-sustained ventricular tachycardia, or the occurrence of sustained ventricular tachycardia not present before drug treatment. Those who required pacemaker implantation because of a sick sinus syndrome or His-bundle ablation were also noted. Overall mortality as well as cardiovascular mortality were recorded.

STATISTICAL ANALYSIS

A two-sided probability level $<0.05$ was regarded as indicating statistical significance. To compare clinical characteristics associated with long-term maintenance of sinus rhythm we used a chi-square test, Wilcoxon-MannWhitney test, or Student's $t$ test if appropriate. Cumulative rates for the time to recurrence of atrial flutter were estimated by Kaplan-Meier methods. Cumulative rates for the time to an event in the groups were compared by the log rank statistic. We used the Cox proportionalhazards regression analysis to determine variables related to maintenance of sinus rhythm after cardioversion(s). Only covariates with $\mathrm{P}$ values $\leqslant 0.20$ in the univariate analysis were entered in this model. Variables modelled as continuous were assessed by determining the quartiles of their distribution, then coefficients for each quartile were determined. Where there was a linear trend of the estimated coefficients of the different groups, the variable was introduced as continuous. If no linearity could be demonstrated the variable was categorised by taking together the quartiles with similar coefficients. The inclusion date was the day of the first cardioversion and the period ended on the day of death, or at the end of December 1993. Follow up after the last cardioversion was at least six months. The analysis was performed using Statistical Analysis System version 6.10 (SAS Institute, Cary, NC, USA).

\section{Results}

\section{OUTCOME OF A SINGLE CARDIOVERSION}

Mean (SD) follow up of all patients was 3.5 (1.7) years (range $0 \cdot 1-7$ years). The baseline characteristics are listed in table 1 . The first electrical cardioversion was successful in 48 patients $(96 \%)$. The remaining two patients underwent a recardioversion after pretreatment with amiodarone. This was successful in one. The actuarial cumulative percentages of patients maintaining sinus rhythm after a single cardioversion, without prophylactic antiarrhythmic drugs being used, was $53 \%, 47 \%$, and $42 \%$ after $0.5,1$, and 5 years, respectively (fig 2, lower line). Univariate and multivariate analysis showed that only increased left atrial size was related to failure of the single shock approach (table 1). Patients with a left atrial size exceeding the median value $(42.5 \mathrm{~mm})$ had a 2.5 risk ratio $(95 \% \mathrm{CI} 1.1$ to $5.7, \mathrm{P}=$ $0.025)$ for failure of the single cardioversion therapy.

OUTCOME OF SERIAL CARDIOVERSION THERAPY Figure 2 shows the percentage of patients who maintained sinus rhythm after serial cardiover- 


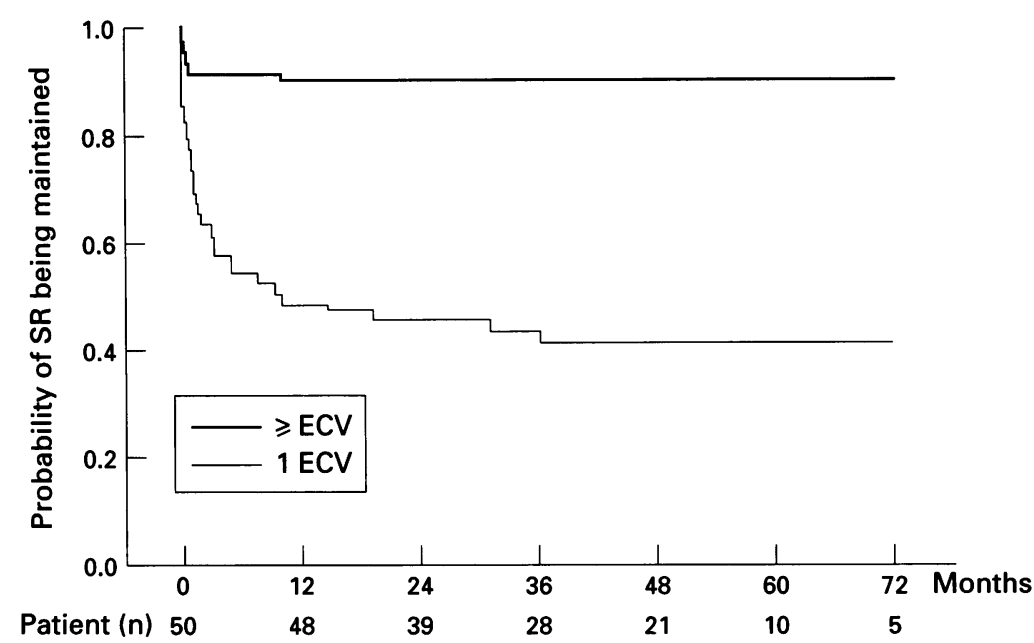

Figure 2 Kaplan-Meier curves showing the probability of maintenance of sinus rhythm (SR) after serial electrical cardioversions (ECV) (upper line) compared with a single shock, no drug approach (lower line). The lower curve shows a steep drop in the beginning mainly because of early relapses. Thereafter there is a gradual decline. The numbers below the figure indicate the numbers of patients at risk during serial cardioversion therapy.

sions. The actuarial cumulative percentages of patients maintaining sinus rhythm was $92 \%$, $90 \%$, and $90 \%$ after $0.5,1$, and 5 years, respectively. Long-term maintenance of sinus rhythm was achieved in 45 patients after a median of two cardioversions (range 1-6) and 29 of these patients responded to the serial cardioversion approach without antiarrhythmic drug therapy. Twenty two of these patients were successfully treated with one cardioversion and seven patients with two cardioversions, respectively. Five patients maintained sinus rhythm on sotalol; two after two and three after three cardioversions, respectively. Two patients were successfully treated with flecainide, both after three cardiover-

Table 2 Major cardiovascular events during follow up

\begin{tabular}{lc}
\hline Event & $n(\%)$ \\
\hline Congestive heart failure (progression): & $5(10)$ \\
Valve surgery & $1(2)$ \\
Adverse events related & $5(10)$ \\
to antiarrhythmic drugs & $2(4)$ \\
Sick sinus syndrome and pacemaker & $10(20)$ \\
Death: & $5(10)$ \\
Sudden & $3(6)$ \\
Progression of heart failure & $2(4)$ \\
Non-cardiovascular & \\
\hline
\end{tabular}

sions. Finally, nine patients maintained sinus rhythm on amiodarone; seven after three, one after five, and one after six cardioversions, respectively. There were no differences in baseline characteristics between patients in whom a serial cardioversion approach was successful and those in whom it failed. However, there was a strong tendency for arrhythmia to have lasted longer (median value 9 v 2.5 months, $P=0.07$ ) and for left ventricular function to be more depressed (mean fractional shortening 25 (7) v $31(7) \%, P=0.09)$ in the patients in whom our serial electrical cardioversion approach failed.

\section{CARDIOVASCULAR EVENTS}

Table 2 lists the cardiovascular events during long-term follow up. Five serious adverse events were related to antiarrhythmic drug treatment and led to termination of medication. Three of five patients who experienced such an event used flecainide; symptoms included visual disorders, intraventricular conduction disturbances, and a significant increase in the number of ventricular premature beats, the latter being classified as proarrhythmia. Sotalol had to be withdrawn in one patient because of exacerbation of reversible airflow obstruction and one patient had severe skin photo-allergy while taking amiodarone. Two patients required cardiac pacing because of sick sinus syndrome. Both patients had had surgery for an atrial septum secundum defect. During follow up 10 patients (20\%) died. Eight deaths were of cardiovascular origin; five were sudden and three were the result of progression of heart failure (table 3). Most of these patients were in sinus rhythm after a single cardioversion but two patients had had three and five cardioversions. These two patients died while they were being treated with amiodarone. Both patients had severe heart failure caused by coronary artery disease. The patient who died in atrial flutter had had two cardioversions. The second cardioversion, during amiodarone treatment, led to two days of sinus rhythm, then the flutter recurred. At that time amiodarone was withdrawn, one month later she died of progression of congestive heart failure. Neither thromboembolic complications nor bleeding complications were encountered during long-term follow up.

Table 3 Characteristics of the cardiovascular deaths

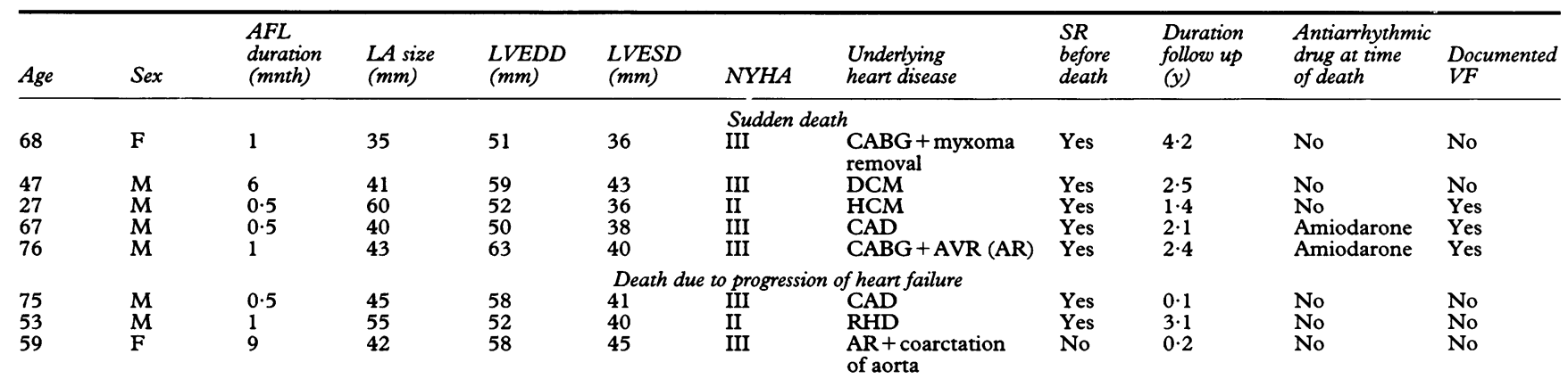

$\overline{\mathrm{AFL}}$, atrial flutter; AR, aortic regurgitation; AVR, aortic valve replacement; CABG, coronary artery bypass surgery; CAD, coronary artery disease; DCM, idiopathic dilated cardiomyopathy; HCM, hypertrophic cardiomyopathy; LA, left atrial; LVEDD, left ventricular end diastolic diameter; LVESD, left ventricular end systolic diameter; NYHA, New York Heart Association class for exercise tolerance; RHD, rheumatic heart disease; SR, sinus rhythm; VF, ventricular fibrillation. 


\section{Discussion}

There are only a few studies of the long-term outcome after electrical cardioversion of chronic atrial flutter. Several of these reports dealt exclusively with young patients who underwent surgical treatment for congenital heart disease. ${ }^{318}$ In these studies antiarrhythmic drug therapy not electrical cardioversion was evaluated. Drug treatment was successful in a few of these patients. Electrical cardioversion was reported to have a high success rate in atrial flutter patients ${ }^{12}$ but this has never been demonstrated for long-term arrhythmia suppression. Most earlier studies in adults were predominantly of patients with atrial fibrillation. ${ }^{214151920} \mathrm{Up}$ to now, no separate data on long-term maintenance of sinus rhythm after cardioversion of adult patients with atrial flutter have been reported. In our long-term study we found that most patients with chronic atrial flutter responded favourably to an electrical cardioversion strategy. After a single shock and without any antiarrhythmic drug treatment, $42 \%$ of the patients maintained sinus rhythm in the long term. The serial cardioversion approach kept up to $90 \%$ of the patients in sinus rhythm during long-term follow up after a median of two cardioversions.

We did not study how long patients should be treated with antiarrhythmic agents during long-term maintenance of sinus rhythm. However, we believe that after two to three years maintenance of sinus rhythm it is likely that the dosage of the antiarrhythmic drug can be reduced and, subsequently that the drug may be withdrawn completely. This issue needs investigation.

Cardiovascular events were common. Symptoms of congestive heart failure were related to progression of the underlying disease. During follow up there were cardiovascular deaths in eight patients. Seven of these patients were in sinus rhythm shortly before death and only two patients were being treated with antiarrhythmic drugs (amiodarone). We believe that this reflects the importance of the underlying disease, in particular heart failure, rather than the arrhythmia in the prognosis of these patients. There may have been other factors in these sudden deaths: (a) a relapse of the arrhythmia complicated by $1: 1$ atrioventricular conduction and subsequent ventricular arrhythmias may have occurred ${ }^{21}$ and (b) proarrhythmia (torsade de pointes) in the two patients who were on amiodarone cannot be excluded. ${ }^{22}$ Garson et al also reported a high incidence of sudden death but this occurred predominantly in patients who were refractory to treatment of the arrhythmia. ${ }^{18}$ In contrast, our patients who died suddenly were probably in sinus rhythm shortly before death. No thromboembolic complications were encountered during long-term follow up when this anticoagulation strategy was used. When the present study started there were no data on the risk of thromboembolic complications in patients with atrial flutter and we decided to treat these patients as if they had atrial fibrillation. Now there are numerous studies indicating the importance of adequate antico- agulation before and after cardioversion of patients with atrial fibrillation. However, data on patients with atrial flutter have not been published. Recently, Jordaens et $a^{23}$ showed atrial dysfunction after cardioversion of atrial flutter; this suggests that atrial flutter may have a comparable impact on atrial function as atrial fibrillation. This finding may support our anticoagulation strategy but more data on thromboembolic complications in patients with atrial flutter are necessary.

In the past few years, interest in the radiofrequency ablation of atrial flutter has increased because success rates are encouraging, especially in patients without underlying heart disease. ${ }^{7-11}$ The acute success rate was $90 \%$ and recurrence rates ranged from 15 to $40 \%$, depending on the duration of follow up and the severity of the underlying heart disease. At first glance, these data suggest that radiofrequency ablation is more sucessful than serial electrical cardioversion. However, the best long-term results of radiofrequency ablation were obtained in patients without underlying heart disease. Only $14 \%$ of our patients showed lone atrial flutter and therefore they resembled the patients included in the ablation studies. In our study the (sudden) death rate was high, whereas in the ablation studies it was low. This result, however, probably merely reflects the short follow up and the absence of patients with severe underlying disease in the ablation study.

In multivariate analysis left atrial size was the only variable that predicted long-term maintenance of sinus rhythm after a single cardioversion of atrial flutter. A left atrial dimension of $\geqslant 42.5 \mathrm{~mm}$ in a parasternal long axis view was associated with an increased risk of the arrhythmia recurring. Univariate analysis showed that those who had had the arrhythmia for longer and those with (echocardiographically determined) decreased left ventricular function were at a slightly increased risk of the serial cardioversion strategy failing. The lack of statistical significance may be caused by the low number of failures.

\section{IMPLICATIONS OF THE STUDY}

This study is the first to demonstrate a favourable arrhythmia outcome in patients with chronic atrial flutter treated with the serial electrical cardioversion strategy. Almost half of the patients were cured after a single shock without antiarrhythmic drug treatment. Therefore such a policy is recommended. The shock should be preceded by echocardiography. This diagnostic procedure gives valuable information on the left atrial dimensions and left ventricular performance, which both help to predict outcome in terms of recurrence of the arrhythmia and the chance of other cardiovascular events and death, respectively. The serial cardioversion approach kept $90 \%$ of the patients in sinus rhythm during long-term follow up. We support this strategy because sinus rhythm is likely to lead to the best pump function and avoids treatment with anticoagulants.

Despite suppression of the arrhythmia, however, cardiovascular mortality was high, 
predominantly in patients with severe structural heart disease. Optimal treatment of the latter is of course necessary. The current position of radiofrequency ablation in atrial flutter is uncertain. Whether the reported high success rate is explained by the elimination of the underlying arrhythmia substrate, the shorter follow up, or a different study population remains to be investigated.

1 Lown B. Electrical cardioversion of cardiac arrhythmias. $\mathrm{Br}$ Heart f 1967;29:469-87.

2 Van Gelder IC, Crijns HJ, Van Gilst WH, Verwer R, Lie KI. Prediction of uneventful cardioversion and maintenance of sinus rhythm from direct-current electrical cardioversion of chronic atrial fibrillation and flutter. $A m \mathcal{F}$ Cardiol 1991;68:41-6.

3 Balaji S, Johnson TB, Sade RM, Case CL, Gilette PC. Management of atrial flutter after the Fontan procedure. $\mathscr{F}$ Am Coll Cardiol 1994;23:1209-15.

4 Waldo AL, McLean WAH, Karp RB, Kouchoukos NT, James TN. Entrainment and interruption of atrial flutter with atrial pacing. Studies in man following open heart surgery. Circulation 1977;56:737-45.

5 Cosio FG, Arribas F, Palacios J, Tascón J, López-Gil M Fragmented electrocardiograms and continuous electrical activity in atrial flutter. $\mathrm{Am} f$ Cardiol 1986;57: 1309-14.

6 Cosio FG, Arribas F, Barbero JM, Kallmeyer C, Goicolea A. Validation of double spike electrocardiograms as markers f conduction delay or block in atrial flutter. $A m \mathcal{F ~ C a r d i o l}$ 1988;61:775-80.

7 Kirkorian G, Moncada E, Chevalier P, Canu G, Claudel J-P, Bellon C, et al. Radiofrequency ablation of atrial flutJ-P, Bellon C, et al. Radiofrequency ablation of atrial flutter. Efficacy of an anatomic

8 Philippon F, Plumb VJ, Epstein AE, Kay N. The risk of atrial fibrillation following radiofrequency catheter ablation of atrial flutter. Circulation 1995;92:430-5.

9 Poty H, Saoudi N, Aziz AA, Nair M, Letac M. Radiofrequency catheter ablation of type 1 atrial flutter. Prediction of late success by electrophysiological criteria. Circulation 1995;92:1389-92.

10 Fischer B, Haissaguerre M, Garrigues S, Poquet F, Gencel $\mathrm{L}$, Clementy J, et al. Radiofrequency catheter ablation of common atrial flutter in 80 patients. $₹ \mathrm{Am}$ Coll Cardiol 1995;25:1365-72.
11 Cosio FG, Arribas F, Lopez-Gil M, Gonzalez HD Radiofrequency ablation of atrial flutter. $\mathcal{F}$ Cardiovasc Electrophysiol 1996;7:60-70.

12 Puech P, Latour H, Grolleau R. Le flutter et ses limites Arch Mal Coeur 1970;63:116-40.

13 Wells JL Jr, MacLean WAH, James TN, Waldo AL Characterization of atrial flutter. Studies in man after open heart surgery using fixed atrial electrodes. Circulation 1979;60:665-73.

14 Crijns $H J$, Van Gelder IC Van Gilst $W H$, Hillege $H$, Gosselink ATM, Lie KI. Serial antiarrhythmic drug Gosselink ATM, Lie Kinus rhythm after electrical cartreatment to maintain sinus rhythm after electrical carAm ₹ Cardiol 1991;68:335-41.

15 Gosselink ATM, Crijns HJ, Van Gelder IC, Hillege H, Wiesfeld ACP, Lie KI. Low-dose amiodarone for maintenance of sinus rhythm after cardioversion of atrial fibrillation or flutter. $\mathcal{F A M A}$ 1992;267:3289-93.

16 Schiller NB, Shah PM, Crawford M, DeMaria A, Devereux R, Feigenbaum H, et al. Recommendations for quantification of the left ventricle by two-dimensional echocardiography. Committee on standards. Subcommittee on quantification of two-dimensional echocardiograms. F Am Soc Echocardiogr 1989;6:358-67.

17 The Cardiac Arrhythmia Suppression Trial (CAST) Investigators. Preliminary report: effect of encainide and flecainide on mortality in a randomized trial of arrhythmia suppression after myocardial infarction. $N$ Engl $f$ mia suppression after

18 Garson A Jr., Bink-Boelkens M, Hesslein PS, Hordof AJ, Keane JF, Neches WH, et al. Atrial flutter in the young: collaborative study of 380 cases. $₹ \mathrm{Am}$ Coll Cardiol 1985;6:871-8.

19 Antman EM, Beamer AD, Cantillon C, McGowan $N$, Goldman L, Friedman PL. Long-term oral propafenone therapy for suppression of refractory symptomatic atrial fibrillation and atrial flutter. $\mathcal{F} \mathrm{Am}$ Coll Cardiol 1988; 12:1005-11.

20 Chun SH, Sager PT, Stevenson WG, Nademanee K, Middlekauff HR, Singh BN. Long-term efficacy of amiodarone for the maintenance of normal sinus rhythm in patients with refractory atrial fibrillation or flutter. $A m \mathcal{F}$ Cardiol 1995;76:47-50.

21 Murgatroyd FD, Camm AJ. Atrial arrhythmias. Lancet 1993;341:1317-22

22 Kopelman HA, Horowitz LN. Efficacy and toxicity of amiodarone for the treatment of supraventricular tachyarrhythmias. Prog Cardiovasc Dis 1989;31:355-66.

23 Jordaens L, Missault L, Germonpré E, Callens B, Adang L, Vandenbogaerde J, Clement DL. Delayed restoration of atrial function after conversion of atrial flutter by pacing or electrical cardioversion. Am 7 Cardiol 1993;71:63-7. 